\title{
Cerebral Cavernous Malformation
}

National Institute of Neurological Disorders and Stroke (NINDS)

\section{Source}

National Institute of Neurological Disorders and Stroke (NINDS). Cerebral Cavernous

Malformation Information Page.

Cerebral cavernous malformations (CCMs) are vascular lesions comprised of clusters of tightly packed, abnormally thin-walled small blood vessels (capillaries) that displace normal neurological tissue in the brain or spinal cord. The vessels are filled with slowmoving or stagnant blood that is usually clotted or in a state of decomposition. Cavernous malformations can occur in the brain, spinal cord, and some other body regions. In the brain and spinal cord these cavernous lesions are quite fragile and are prone to bleeding, causing hemorrhagic strokes (bleeding into the brain), seizures, and neurological deficits. CCMs can range in size from a few fractions of an inch to several inches in diameter, depending on the number of blood vessels involved. Some people develop multiple lesions while others never experience related medical problems. Hereditary forms of CCM are caused by mutations in one of three CCM disease genes: CCM1, CCM2, and CCM3. A large population with hereditary CCM disease is found in New Mexico and the Southwestern United States, in which the disease is caused by mutations in the gene CCM1 (or KRIT1). 